\title{
La perspectiva de estudiantes en línea sobre los entornos virtuales de aprendizaje en la educación superior
}

\section{A Online students' perspective about virtual learning environment in higher education}

\author{
Alfredo Blanco Martínez ${ }^{1}$, Pilar Anta Fernández ${ }^{2}$ \\ ${ }^{1}$ Departamento de Didáctica y Organización Escolar, Universidad de A Coruña, España (hisabm01@udc.es) \\ ${ }^{2}$ Departamento de Didáctica y Organización Escolar, Universidad de A Coruña, España (pilar.anta@udc.es)
}

Recibido el 28 de noviembre de 2015; revisado el 19 de septiembre de 2016; aceptado el 27 de octubre de 2016; publicado el 2 de diciembre de 2016

\section{RESUMEN:}

El proceso de enseñanza-aprendizaje se encuentra constantemente en una fase de evolución, debido a los cambios e innovaciones que surgen en la sociedad. Esta transformación tiene como objetivo responder a las demandas educativas de los ciudadanos, ya que estos exigen nuevas vías y caminos para continuar aprendiendo $\mathrm{y}$, al mismo tiempo, para enriquecer su formación a lo largo de la vida. En este contexto, ocupan un lugar importante las tecnologías de la información y de la comunicación (TIC) y, concretamente, los entornos virtuales de aprendizaje (EVA). En la educación superior, los EVA se configuran como espacios virtuales de carácter educativo para la formación individual y colectiva de los usuarios en un nivel inicial, continuado y permanente. Por este motivo, interesa conocer el grado de aceptación de los estudiantes que participan de este modelo de enseñanza y aprendizaje.

En este trabajo, se examina y describe la percepción de un grupo de alumnos de la Universidad Autónoma de Barcelona, con el fin de identificar los beneficios y dificultades que presentan estos espacios en los estudios de posgrado. Para ello, se ha llevado a cabo una investigación cualitativa, en la que cobran vida la narrativa de las estudiantes y sus experiencias en los EVA. En este caso, se ha utilizado la entrevista como técnica recogida de datos, con el propósito de atender de una forma individualizada a las concepciones y opiniones de cada una de las participantes. Tras el análisis y comentario de los resultados, cabe subrayar que los EVA son una posibilidad abierta para la formación de las personas a través de un modelo de enseñanza basado en la flexibilidad, la eficacia, la adecuación y la rigurosidad.
PALABRAS
CLAVE:
ENSEÑANZA, ENTORNOS VIRTUALES DE APRENDIZAJE, TIC, EDUCACIÓN SUPERIOR, INVESTIGACIÓN CUALITATIVA.

\begin{abstract}
:
The teaching-learning process is constantly in a state of evolution, due to the changes and innovations that arise in society. This transformation has the aim to meet the educational demands of citizens, because they require new ways to improve their knowledge and, at the same time, to enrich their lifelong learning. In this context, Information and Communication Technologies (ICT) have an important place in the society and, specifically, the virtual learning environments (EVA). In higher education, the EVA is configured as virtual educational spaces for individual and collective training of users in an initial, continuous and permanent level. For this reason, we want to know the degree of acceptance of the students participating in this model of teaching and learning.

In this paper, we examine and describe the perception of a group of students of University of Barcelona, in order to identify the benefits and difficulties presented by these spaces in postgraduate studies. For do this, we have carried out a qualitative
\end{abstract}


study, in which we have considered the narrative of the students and their experiences in the EVA. In this case, we have used the interview as data collection technique, in order to attend to the individual opinion of each participant. After analysis data and discussion of the results, we can emphasize that the EVA are a possibility to the people education through a teaching model based on flexibility, efficiency, adequacy and preciseness.

KEYWORDS: TEACHING, VIRTUAL LEARNING ENVIRONMENT, ICT, HIGHER EDUCATION, QUALITATIVE RESEARCH.

\section{INTRODUCCIÓN}

El mundo en el que vivimos se ha ido transformando e innovando de forma vertiginosa en los últimos años. Los cambios surgidos en la sociedad han ido generando nuevas formas de producción, de relacionarse y de vivir, configurando lo que se ha dado en llamar la sociedad del conocimiento. En este sentido, hay que reconocer que la mejora de los procesos de comunicación, relación y aprendizaje en la sociedad actual ha sido posible gracias al papel protagonista de las TIC.

Asumiendo la conceptualización de Cobo Romaní (2009) entendemos las TIC como innovaciones "que permiten el procesamiento y acumulación de enormes cantidades de información, además de una rápida distribución de la información a través de redes de comunicación" (p. 305). Tal y como señalan Sánchez Asín, Boix y Jurado de los Santos (2009), hay que tener en cuenta las herramientas tecnológicas disponibles y otras que puedan aparecer para mejorar los modelos de enseñanza-aprendizaje, así como replantearse las prácticas docentes (Cabero, 2010; González Sanmamed, 2007; Sangrà y González-Sanmamed, 2010).

En este contexto, nacen los EVA como una forma de transformar los entornos educativos tradicionales para crear nuevos escenarios de enseñanza y aprendizaje virtual o en línea al servicio de toda la comunidad educativa (Bustos y Coll, 2010).

\subsection{Los EVA en la Educación Superior}

La Universidad tiene un fuerte compromiso con la sociedad, ya que su objetivo es construir profesionales y especialistas, crear conocimiento, fabricar ideas y ser un crisol cultural multidisciplinar (Canay Pazos, 2008). En este sentido, se ha constituido como uno de los ejes centrales entre TIC, educación y formación.
De acuerdo con Hernández Sellés (2015), las TIC han irrumpido en la Educación Superior como herramientas de apoyo en los procesos de enseñanza-aprendizaje. Así, se han configurado distintos recursos digitales y entornos virtuales para la formación de estudiantes y otros miembros de la comunidad educativa.

En línea con Khan (2005), López, Flores, Rodríguez y De la Torre (2012), las universidades han apostado por una serie de dimensiones para que las plataformas virtuales posean un sello de calidad y ofrezcan garantías a los estudiantes. Dichas dimensiones son:

- Institucional a nivel organizativo y académico.

- Gestión de los procesos.

- Tecnológica a nivel infraestructura.

- Pedagógica.

- Ética.

- Diseño de la interfaz.

- Apoyos y soportes de ayuda.

- Evaluación.

Concretamente, en lo que respecta a los EVA, se trata de sistemas de gestión del conocimiento que apuestan por la formación continuada de las personas a través de una plataforma virtual. Según Silva (2011) se configuran como espacios con finalidades formativas, sociales $y$ activas que enriquecen tanto el aprendizaje virtual como el presencial. Además, integran un multiperspectivismo tecnológico y pedagógico. Teniendo en cuenta las aportaciones de Belloch (2012) estos espacios poseen las siguientes características:

- Acceso protegido (usuario y contraseña).

- Disponibilidad de herramientas web 1.0 y 2.0.

- Diseño gráfico intuitivo y de fácil comprensión.

- Estructura organizada por bloques y secciones temáticas, módulos teóricos, actividades, evaluación, etc.

- Adaptados al usuario y a sus necesidades.

- Seguimiento garantizado por el tutor, los profesores de los módulos y el administrador.

- Comunicación e interacción instantánea.

- Espacio creado para el almacenamiento e intercambio de información.

Paralelamente, Boneu (2007) fundamenta las características de los EVA en los siguientes pilares: interactividad, flexibilidad, escalabilidad y estandarización. En suma, se trata de un espacio didáctico creado para la asincronía, la planificación y la organización, la agrupación, la comunicación y 
el trabajo colaborativo (Bautista, Borges y Forés, 2006).

En relación con las premisas teóricas, en este trabajo interesa conocer qué piensan los estudiantes sobre estos entornos virtuales, con el fin de seguir trabajando en la mejora de los procesos de enseñanza-aprendizaje.

\section{METODOLOGÍA DE INVESTIGACIÓN}

La investigación cualitativa permite al investigador acercarse a cualquier hecho o fenómeno en acción desde una óptica real, científica y educativa. De acuerdo con Flick (2015), esta metodología responde interrogantes generales y específicos, al mismo tiempo que averigua la arquitectura exterior e interior del objeto de estudio y su funcionamiento en un contexto social determinado. En suma, se trata de un método a través del cual se realiza un viaje interior a un mundo en el que la observación y la comprensión son la clave para generar conocimiento.

\subsection{Objetivos}

El presente trabajo ha tenido el propósito de averiguar y analizar la perspectiva de estudiantes de educación superior sobre los entornos virtuales de aprendizaje. Asimismo, se han contemplado los siguientes objetivos específicos:

- Descubrir la concepción que tienen los estudiantes sobre los EVA.

- Conocer qué opina el alumnado sobre los EVA en la educación superior.

- Identificar las motivaciones que les han llevado a participar en un espacio virtual de aprendizaje.

- Reconocer en el discurso de los participantes los principales beneficios y/o dificultades de los EVA en la educación superior.

\subsection{Participantes}

Han colaborado en esta investigación un total de cuatro alumnas que cursan un estudio de posgrado oficial en TIC y en educación, en una Universidad privada de la Comunidad Autónoma de Barcelona. Dicha institución oferta titulaciones en modalidad online de grado, posgrado, especializaciones $\mathrm{y}$ estudios de tercer ciclo de la rama científica, tecnológica, humanística y de las ciencias sociales y jurídicas.

En esta titulación, el número total de estudiantes matriculados es de $80 \quad(n=80)$. En este caso, la selección de los participantes no ha sido aleatoria, sino que contamos con la ayuda de un "portero" para entrar en contacto con un grupo de alumnas interesadas en participar en este proyecto.
Concretamente, han contribuido con sus testimonios cuatro mujeres de edades comprendidas entre $\operatorname{los} 25$ y 50 años que ejercen, actualmente, como docentes en la Educación Secundaria. Para tres de las alumnas es la primera vez que se forman a través de un espacio virtual de aprendizaje. No obstante, hay una estudiante que lleva más de tres años al servicio de los EVA. Todas ellas se han visto motivadas a inscribirse en estudios de estas características por causas familiares y/o laborales, con el propósito de seguir formándose y actualizándose como profesoras, así como para futuras oportunidades profesionales.

\subsection{Técnica de recogida de datos}

Una forma de entrar en contacto con el mundo del investigado es a través de la entrevista. En un sentido lingüístico, se trata de un intercambio a dos voces entre el entrevistador y el entrevistado en un marco y situación concreta. Entonces, surge una pieza musical por la que desfilan los agudos y graves y en dónde cobran significado las palabras. Por su parte, como técnica de recogida de datos permite conocer en profundidad la visión del investigado sobre un tema o fenómeno determinado (González Sanmamed, 1994; Kvale, 2011).

En lo que respecta a este trabajo, la entrevista se ha utilizado con el fin de averiguar qué opinan los estudiantes de los EVA y sobre esta modalidad de enseñanza y aprendizaje. De esta manera, a través de sus aportaciones hemos podido dotar de conocimiento a la comunidad científica y, de igual manera, caminar hacia la mejora y calidad educativa en la educación superior (Valles, 2009).

Atendiendo a Simons (2011), hemos empleado la entrevista de tipo cualitativo, o también denominada en profundidad, y estructurada. En este caso, se aplicó una única entrevista a cada una de las participantes y de forma individualizada. La decisión de llevar a cabo una única entrevista se debe, fundamentalmente, para ajustarse a la disponibilidad horaria de las informantes. Dicha entrevista se estructuró en tres bloques de contenidos con un total de 25 preguntas. Se ha querido tener sesiones individuales y únicas con cada investigada, con el propósito de lograr un clima de confianza, así como una comunicación e intercambio de información fluida. Así, se evitó que ninguna voz cobrara mayor protagonismo que otra (Wolf, 2012).

Con el propósito de mantener el anonimato en la exposición de los resultados, se utilizarán abreviaturas (E) para referirnos a cada una de las entrevistadas y, también, se les asignará un número de identificación para organizar correctamente los discursos de las informantes (E1, E2, E3, E4). 


\subsection{Análisis e interpretación de la información}

De acuerdo con Gibbs (2012), es necesario organizar, estructurar y codificar los datos recogidos para realizar el correspondiente informe y la consiguiente exposición de los resultados. Para ello, hay que discernir de las narraciones de los entrevistados aquella información que responda a los objetivos del trabajo (Rapley, 2014). En este caso, se ha tomado como referente el principio de análisis de Miles y Huberman (1994) centrado en la reducción, exposición, verificación y conclusión de los datos.

Finalmente, este trabajo se ha estructurado en torno al esquema de análisis DAFO para profundizar a nivel interno y externo (figura 1).

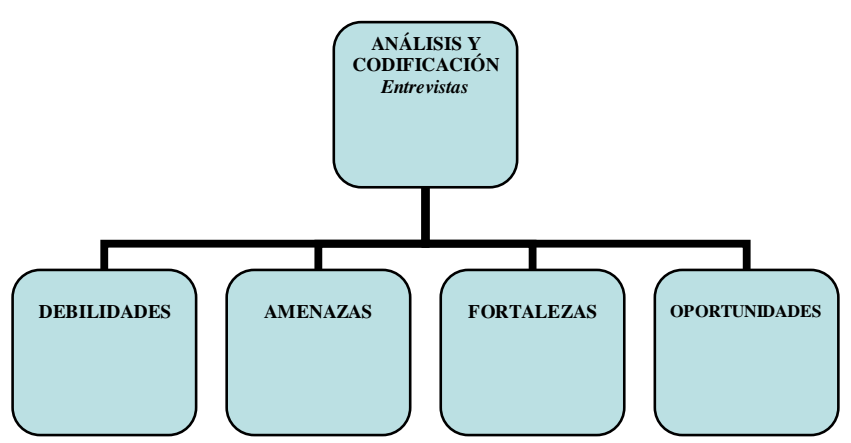

Figura 1. Esquema de codificación y análisis de los datos

\section{RESULTADOS}

Para la exposición de los resultados hemos tenido en consideración el esquema de análisis DAFO, ya que pone de relieve aquellos puntos débiles y fuertes en torno a los EVA. Todas las participantes coinciden en que se trata de un espacio web para el aprendizaje, el cual se vale de distintas herramientas digitales para llevar a cabo las acciones formativas. No obstante, como veremos a continuación, las investigadas también nos ofrecen una mirada crítica y personal para poner de relieve los beneficios y desventajas de estos espacios en la educación superior.

\subsection{Debilidades}

Bajo el rótulo "debilidades" se recogen aquellos puntos que son necesarios transformar para la mejora de la calidad educativa de los EVA. Por un lado, la entrevistada A manifiesta que son espacios poco aprovechados y "quizás puede ser debido a que una buena parte del profesorado no tiene interés en formarse para sacar el máximo provecho" (E1, p. 1). En este sentido, la estudiante C nos comenta que "sería interesante que se ofertara una mayor formación en EVA entre los profesionales de la educación, con el fin de que ofrecieran un modelo de aprendizaje virtual más generalizado y complementario a los estudios en formato presencial" (E3, p. 6). Tal y como señala la alumna A "esto se consigue mediante formación específica para los docentes y creando programas más intuitivos y prácticos" (E1, p. 2).

Por otra parte, la participante A subraya como debilidad "la posible dependencia que puede crearse en la sociedad por las TIC y sus respectivos programas y aplicaciones" (E1, p. 3). Por este motivo, nos sugiere que "las personas deben comprender que los entornos virtuales son espacios al servicio de los contextos reales de aprendizaje y, por tanto, no conllevan a la deshumanización de la educación" (E1, p. 4). En suma, "se trata de que aprendamos a convivir, a aprender y a trabajar en ambos mundos" (E1, p. 5).

Otro punto de interés, es el que nos ofrece la estudiante B vinculado a la desorganización de los EVA. Por ello, nos aporta que "dichos espacios también deben fomentar la interacción y las relaciones entre alumnado y profesor, así como los grupos de trabajo" (E2, p. 2). Por otro lado, también considera que "el material que nos presentan es demasiado extenso y tedioso y, por eso, creo que podría resumirse" (E2, p. 2). De la misma manera, la alumna $\mathrm{C}$ cree que "los materiales para el alumnado resultan monótonos y repetitivos, lo que hace que perdamos más tiempo" (E3, p. 2). Por ello, entiende que "la comunicación entre profesorado debe ser igual de fluida que en el ámbito presencial, con el objetivo de no reiterar las mismas ideas en las lecturas propuestas para el alumnado" (E3, p. 4).

En último lugar, la estudiante $\mathrm{D}$ focaliza las debilidades en los usuarios. Ella piensa que "parte del trabajo de los EVA y de sus implicados es moderar y motivar a los alumnos/as, pero cuando no ocurre el entorno se ve afectado por la falta de eficiencia y conlleva un empobrecimiento en el proceso de enseñanza-aprendizaje del estudiante" (E4, p. 2). Por esta razón, apuesta "por una motivación sustentada en las prácticas formativas, en las actividades, en los datos teóricos, así como en el material complementario" (E4, p. 5).

Teniendo en cuenta el testimonio de las investigadas, podemos identificar las siguientes debilidades:

- Falta de formación específica en TIC, en su metodología y didáctica entre el corpus docente.

- La ausencia de interacción y comunicación entre el profesorado participante.

- La sobrecarga informativa y poco rentable para un aprendizaje con éxito.

- El bajo nivel de motivación de los usuarios. 


\subsection{Amenazas}

En lo que respecta a los posibles peligros derivados de los EVA, las entrevistadas reconocen aquellos relacionados con los destinatarios, el diseño de la plataforma y las concepciones de los individuos. Con respecto al primer punto, la alumna A piensa que las amenazas "se pueden encontrar en otros niveles de enseñanza, como en educación primaria y secundaria, debido a los contenidos a los que los menores de edad puedan acceder" (E1, p. 7). En la misma línea, expone sus argumentos la investigada D. Esta última, afirma que "sería necesario un sistema de control de acceso y de vigilancia mayor para todos los niveles, ya que pueden surgir problemas en la relación del grupo-clase o, de la misma forma, en la construcción de los conocimientos. Este tipo de acontecimientos provocaría entre los usuarios percepciones erróneas sobre cualquier asunto y/o tema teórico" (E4, p. 10).

De otra parte, la participante $C$ explica que los problemas se concentran en torno a las concepciones sobre los EVA. Esta alumna nos comenta que "muchas personas creen que las enseñanzas virtuales son más fáciles y menos exigentes que las presenciales" (E3, p. 12). Por esta razón, entiende que es vital promover, socialmente, el carácter "exigente y de calidad educativa de los EVA" (E3, p. 11).

Por otra parte, el testimonio de la participante B se concentra en el diseño de los EVA. Es decir, concibe que "los EVA tienen peligro cuando el entorno virtual no está diseñado adecuadamente. Para ello se necesita de un diseño tecno-pedagógico que motive y facilite el proceso de enseñanzaaprendizaje de los estudiantes" (E2, p. 9).

A pesar de haber identificado una serie de riesgos, las investigadas convienen que son de fácil solución, siempre y cuando, en la comunidad haya un interés por la mejora y por los resultados obtenidos. En esta línea, creen que siempre es necesario medir los niveles de satisfacción de los participantes, así como evaluar las sugerencias o recomendaciones que haga el alumnado. En suma, afirman que la inexperiencia y el carácter novedoso de estos espacios, irá creciendo y madurando a través del tiempo y de las distintas experiencias.

\subsection{Fortalezas}

Cuando hablamos de fortalezas, nos referimos a los puntos fuertes de los EVA. En este caso, las participantes lo tienen bastante claro y sus respuestas confluyen en un mismo punto: la flexibilidad. A continuación, mostramos en un cuadro resumen las palabras clave derivadas de las narraciones de las investigadas (tabla 1).
Tabla 1. Posturas en torno a la flexibilidad

\begin{tabular}{|c|l|}
\hline Alumna A & $\begin{array}{l}\text { - Temporal } \\
\text { - Capacidad de } \\
\text { adaptación }\end{array}$ \\
\hline Alumna B & $\begin{array}{l}\text { - Formación } \\
\text { continuada } \\
\text { - Posibilidad de } \\
\text { ritmos }\end{array}$ \\
\hline Alumna C & $\begin{array}{l}\text { - Accesibilidad } \\
\text { - Comunicación }\end{array}$ \\
\hline Alumna D & $\begin{array}{l}\text { - Inmediatez } \\
\text { - Libertad }\end{array}$ \\
\hline
\end{tabular}

Los conceptos destacados en la tabla 1 ponen de manifiesto el carácter altamente pedagógico, no solo de los EVA, sino de cualquier herramienta TIC. Atendiendo al testimonio de la estudiante C, cabe destacar que "la enseñanza a través de los EVA permite nuevas posibilidades que no podemos obviar, por ejemplo, la formación continuada, el reciclaje constante. Algunos profesionales como los médicos, profesores e ingenieros, entre otros, necesitamos de actualización constante y continuada, y este tipo de enseñanza-aprendizaje te permite compaginar la vida laboral y la académica" (E3, p. 16).

Paralelamente, la participante D confirma que son entornos que construyen "una base sólida tanto para la enseñanza-aprendizaje, como para la formación" (E4, p. 13). En este sentido, cree que "es un universo perfecto con aplicaciones en el aula real, con mayor dinamismo y con un feedback constante" (E4, p. 14).

En suma, las principales fortalezas identificadas son las siguientes:

- Comunicación directa e instantánea con el grupo-clase y con los contenidos de aprendizaje.

- Accesibilidad y rapidez para disponer de todo tipo de materiales.

- Intercambiabilidad de opiniones, trabajos e información académica.

- Tiempos de trabajo adaptables a la situación personal y laboral de cada estudiante.

- Organización y distribución temporal flexible y libre.

- Adaptación a las necesidades específicas de cada alumno.

- Posibilidad formativa a lo largo de la vida.

\subsection{Oportunidades}

En cuanto a las oportunidades, cada una de las participantes destaca un punto favorable dependiendo de su situación y marco socio-personal. 
Por un lado, la participante A considera que "los EVA ayudan a que construyamos una identidad tanto personal como académica y, al mismo tiempo, son una ocasión para reciclarnos profesionalmente y, también, para revalorizar conocimientos ya adquiridos junto con la nueva información" (E1, p. 20). Por su parte, la entrevistada B subraya que "la participación en este espacio ha provocado un cambio en mi interior, porque me ha servido para comprender que estos entornos son igual de adecuados para una formación sustentada en la calidad educativa" (E2, p. 22). En la misma sintonía, la alumna D confirma que "los EVA son espacios dignos de admiración dado su carácter polifacético" (E4, p. 19). Además, nos comenta que "el mundo EVA provoca que te impliques personal $y$ socialmente con el resto de usuarios/as, es decir, ha sido un momento para reflexionar sobre mis anteriores experiencias y, así, valorar la importancia de trabajar en equipo respetando y aprendiendo de las aportaciones de los demás" (E4, p. 22).

Con perspectiva de futuro, las participantes consideran de forma unísona que las universidades, tanto públicas como privadas, deben ampliar su oferta de títulos en este formato dadas las infinitas posibilidades formativas y laborales que ofrecen los mismos. En este sentido, la entrevistada C nos comenta que "es mundo lleno de oportunidades que contribuyen a la equidad en la educación. Es un logro que cualquier persona del mundo pueda estudiar en cualquier Universidad de prestigio sin plantearse gastos de transporte, estancia o dietas. En definitiva, es una forma de contribuir a que nuestro mundo sea un lugar mejor" (E3, p. 26).

A pesar de que queda mucho camino por recorrer, las participantes afirman que es un mundo en proceso evolutivo cuyos pilares son: la enseñanza, el aprendizaje, la interacción, la formación y la calidad.

\section{CONCLUSIONES}

En línea con Flick (2015), la investigación cualitativa nos aporta información específica sobre un hecho significativo a nivel científico y humano. En esta ocasión, la entrevista nos ha arrojado luz sobre los recientes EVA, lo que nos ha permitido generar conocimiento para mejorar en la creación, en la organización y en el estudio de estos espacios.

A continuación, teniendo en cuenta los objetivos previamente formulados, exponemos las conclusiones a las que hemos llegado a través de este trabajo:

- Concepción sobre los EVA. Las participantes conciben los EVA como espacios de aprendizaje para la educación y la formación de los individuos. Además, es una herramienta al servicio de docentes y alumnos destinada a realizar aprendizajes más colaborativos y participativos. En suma, entienden que es un espacio virtual creado para poder realizar actividades, introducir materiales, promover áreas de discusión, incluir temáticas, comunicarse e interactuar con los usuarios desde cualquier parte del mundo y en cualquier momento. Por todas estas razones, conciben que es un mundo paralelo en el que los aprendizajes se adquieren en línea recta, de forma continuada y flexible, y en base a la situación particular de cada individuo.

- El papel de los EVA en la educación superior. A través del relato de cada una de las entrevistadas, comprendemos que los EVA se encuentran en pleno auge en la educación superior, debido a la creciente demanda social por continuar formándose a lo largo de la vida. Además, para el aprendizaje virtual el alumnado solo necesita de conexión a Internet y una mínima formación en conocimientos ofimáticos, ya que las plataformas y aplicaciones cada vez son más intuitivas y fáciles de manejar. Por otra parte, también cabe destacar que en dichos espacios la atención es totalmente personalizada $\mathrm{y}$ continuada durante todo el proceso. Las investigadas destacan de sus respectivos tutores su capacidad orientadora, así como su involucración para la resolución de dudas y/o conflictos. No obstante, teniendo en cuenta la experiencia de estas estudiantes, los EVA no han producido el suficiente impacto entre las profesionales de la educación. En ocasiones, la falta de formación y/o el desinterés por involucrarse en proyectos con carácter innovador y transformador, conlleva a que el aprendizaje en los EVA se ralentice y el alumnado sufra las consecuencias. Por ello, es vital que haya un mayor nivel de concienciación y de compromiso entre los docentes participantes $\mathrm{y}$, al mismo tiempo, un hilo comunicativo operativo para el intercambio de experiencias. En definitiva, se trata de conducir a los profesores hacia una ecología de su propio aprendizaje para que se actualicen y preparen en torno a las recientes transformaciones sociales.

- Motivos y/o razones de participación en los EVA. La mayoría coincide en que su participación en los EVA ha sido por cuestiones relativas al tiempo y por su 
situación laboral y/o familiar. No obstante, en sus palabras dejan entrever que cierta grado de curiosidad les ha empujado a ser partícipes de este nuevo mundo. Había en ellas una intención por experimentar y cambiar su rol tradicional como estudiantes. Por otra parte, tal y como nos sugieren las entrevistadas, es primordial que la sociedad le pierda el miedo a lo desconocido. De esta manera, se logrará una expansión creciente de los EVA en un futuro cercano, en el que aprendizaje presencial y virtual, no se discriminen. Por el contrario, se pretende que ambas gocen del mismo prestigio y de la misma calidad. Por tanto, la elección de una u otra modalidad debe estar sujeta a la situación de cada uno de los individuos y/o gustos personales.

- Beneficios y dificultades de los EVA. Como toda forma de aprendizaje, los EVA tienen sus beneficios y dificultades. En ambos casos, hemos intentado relatar en el informe aquellos aspectos que nos han resultado más llamativos. De todos modos y de acuerdo con las investigadas, creemos que el mayor problema es por razones económicas. En la mayoría de los casos, la oferta proviene del sector privado con costes superiores a los públicos, lo que provoca que algunas personas no puedan acceder a este tipo de estudios. Por este motivo, con el objetivo de lograr una educación equitativa y con el mismo derecho de oportunidades, es necesario provocar el despertar de las Universidades públicas, así como de sus respectivas administraciones, para que concentren sus miradas en estos modelos de aprendizaje. En este sentido, hay que causar un mayor impacto para que se apueste por esta modalidad de aprendizaje y se invierta, económicamente, en el cambio y en la innovación educativa.

Con visión de futuro, sería interesante realizar un estudio profundizado y más ambicioso sobre los EVA, con el fin de atender a las necesidades formativas, a las recomendaciones y sugerencias del alumnado, así como a los cambios tecnológicos y pedagógicos subyacentes en la sociedad. De este modo, y comparando las distintas experiencias y percepciones de personas de distintas partes del mundo, podremos avanzar hacia la mejora, haciendo de los EVA espacios respetables y reconocidos universalmente como verdaderas opciones para la educación y la formación de las personas.

\section{DISCUSIÓN}

Los recientes trabajos de investigación de autores como Hernández Sellés (2015); Nóbile y Luna (2015); Castevich, Goodin, Hogan, Sanders, Slovec y Yelizarov (2015), abordan cuestiones centralizadas en los EVA y en sus aplicaciones y finalidades en la educación superior. Estas investigaciones ponen de relieve el carácter innovador, didáctico y pedagógico de dichas plataformas, ya que son una opción inminente para el aprendizaje y la formación desde una óptica renovadora. Se trata de experiencias educativas que apuestan por la formación inicial, continuada y permanente de los ciudadanos en un determinado saber científico, al mismo tiempo que promueven la utilización de las TIC desde una perspectiva constructiva.

A pesar de que los resultados sean positivos y los EVA avancen a una velocidad vertiginosa, hay que centrar los esfuerzos en la mejora y en la calidad de dichos espacios, con el fin de que tanto el docente como el alumno se sientan parte del proceso de enseñanza-aprendizaje sin que exista ninguna barrera que lo pueda impedir. Para ello, es fundamental analizar el grado de satisfacción de alumnos y profesores.

Teniendo en cuenta nuestro trabajo y la experiencia de los participantes, creemos que es fundamental proporcionar una mayor formación en EVA al personal docente que se involucre en este modelo de enseñanza, ya que como dice la frase célebre de Solomon Ortiz "La educación es la clave del éxito en la vida, y los profesores tienen un impacto duradero en las vidas de sus alumnos". En este sentido, consideramos que las distintas instituciones educativas y/o universidades partícipes de este modelo de enseñanza, deben promover una formación rigurosa y actualizada al profesorado. De este modo, se logrará que la formación docente sea permanente y supervisada, "que deben hacerse explícitos los beneficios del uso de las TIC, que deben incluirse aspectos técnicos como pedagógicos, que debe responderse de manera explícita a las necesidades de los profesores y proveer a los profesores de posibilidades de innovación y mejora en su tarea docente" (Monsiváis, McAnally y Lavigne, 2014:94-95).

\section{REFERENCIAS}

Bautista, G., Borges, F. y Forés, A. (2006). Didáctica universitaria en entornos virtuales de enseñanzaaprendizaje. Madrid: Narcea.

Belloch, C. (2012). Entornos virtuales de aprendizaje. Valencia: Universidad de Valencia.

Boneu, J. M. (2007). Plataformas abiertas de e-learning para el soporte de contenidos educativos abiertos. Revista de Universidad y Sociedad del Conocimiento, 4 (1), 36-47. 
Bustos, A. y Coll, C. (2010). Los entornos virtuales como espacios de enseñanza y aprendizaje. Una perspectiva psicoeducativa para su caracterización y análisis. Revista Mexicana de Investigación Educativa, 15 (44), 163-184.

Cabero, J. (2010). Los retos de la integración de las TIC en los procesos educativos: límites y posibilidades. Perspectiva Educacional, 49 (1), 32-61.

Canay Pazos, J. R. (2008). El uso de entornos virtuales de aprendizaje en las Universidades presenciales: Un análisis empírico sobre la experiencia del campus virtual de la USC. (Tesis doctoral inédita). Universidad de Santiago de Compostela.

Castevich, J., Goodin, A. D., Hogan, D. L., Sanders, L., Slovec, K. y Yelizarov, D. (2015). Desarrollo del entorno personal de aprendizaje para tutoría e investigación en niveles educativos superiores. Revista InnoEduca. International Journal of Technology and Educational Innovation, 1, 15-26.

Cobo Romaní, J. C. (2009). El concepto de tecnologías de la información. Benchmarking sobre las definiciones de las TIC en la sociedad del conocimiento. Revista Zer, 14 (27), 295-318.

Flick, U. (2015). El diseño de la investigación cualitativa. Madrid: Morata.

Gibbs, G. (2012). El análisis de datos cualitativos en investigación cualitativa. Madrid: Morata.

González Sanmamed, M. (1994). Aprender a enseñar: mitos y realidades. A Coruña: Servicio de Publicaciones de la Universidad de A Coruña.

González Sanmamed, M. (2007). Las TIC como factor de innovación y mejora de la calidad de la enseñanza. En J. Cabero (Coord.). Tecnología Educativa. (219-232). Madrid: McGrawHill.

Hernández Sellés, N. (2015). El trabajo colaborativo en entornos virtuales en educación superior. (Tesis Doctoral Inédita). Universidad de A Coruña.

Khan, Badrul (2005). Managing E-learning Strategies. Design, Delivery, Implementation and Evaluation. Hershey, pa: Information Science Publishing (Idea Group Inc.)

Kvale, S. (2011). Las entrevistas en investigación cualitativa. Madrid: Morata.

López, M. C., Flores Guerrero, K., Rodríguez Hernández, M. A. y De la Torre García, E. Análisis de una experiencia de entornos virtuales de aprendizaje en educación superior: el programa de cursos en línea del centro universitario del sur de la Universidad de Guadalajara, México. Revista Iberoamericana de Educación, 60, 97-115.

Monsiváis, M.I., McAnally, L. y Lavigne, G. (2014). Aplicación y validación de un modelo tecnopedagógico de formación docente mediante una plataforma educativa virtual. Revista de Universidad y Sociedad del Conocimiento (RUSC), 11 (1), 91-107.

Miles, M.B. y Huberman, A.M. (1994). Qualitative Data Analysis: An expanded sourcebook. Thousand Oaks, California: SAGE.

Nóbile, C. I. y Luna, A. E. (2015). Los Entornos Virtuales de Enseñanza y Aprendizaje en la Universidad Nacional de la Plata. Una aproximación a los usos y opiniones de los estudiantes. Revista InnoEduca. International Journal of Technology and Educational Innovation, 1, 3-9.

Rapley, T. (2014). Los análisis de conversación, de discurso y de documentos en investigación cualitativa. Madrid: Morata.

Sánchez Asín, A., Boix Peinado, J.L. y Jurado de los Santos, P. (2009). La sociedad del conocimiento y las TICS: Una inmejorable oportunidad para el cambio docente. PíxelBit. Revista de Medios y Educación, 34, 179-204.

Sangrà, A. y González-Sanmamed, M. (2010). The role of information and communication Technologies in improving teaching as learning processes in primary and secondary schools. Research in Learning Technology, 18 (3), 207-220.

Silva, J. (2011). Diseño y moderación de entornos virtuales de aprendizaje (EVA). Barcelona: Editorial UOC.

Simons, H. (2011). El estudio de caso. Teoría y práctica. Madrid: Morata.

Valles, M. S. (2009). Cuadernos metodológicos. Entrevistas cualitativas. Madrid: CSI

Wolf, V. (2012). Una habitación propia. Madrid: Alianza Editorial. 